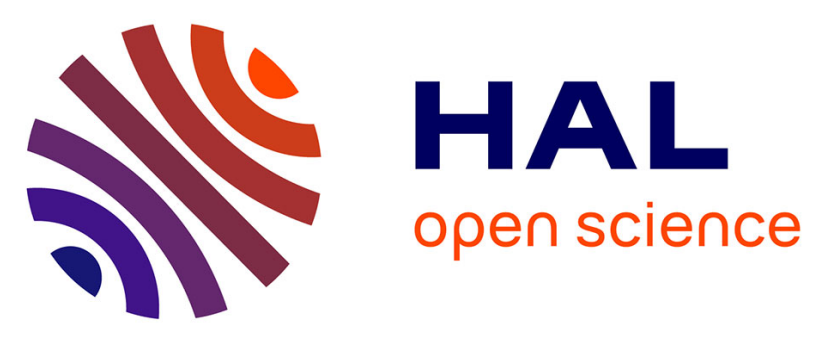

\title{
The clpP multigenic family in Streptomyces lividans: conditional expression of the clpP3 clpP4 operon is controlled by PopR, a novel transcriptional activator
}

Julie Viala, Georges Rapoport, Philippe Mazodier

\section{To cite this version:}

Julie Viala, Georges Rapoport, Philippe Mazodier. The clpP multigenic family in Streptomyces lividans: conditional expression of the clpP3 clpP4 operon is controlled by PopR, a novel transcriptional activator. Molecular Microbiology, 2000, 38 (3), pp.602-612. 10.1046/j.1365-2958.2000.02155.x . hal03269528

HAL Id: hal-03269528

https://hal-amu.archives-ouvertes.fr/hal-03269528

Submitted on 24 Jun 2021

HAL is a multi-disciplinary open access archive for the deposit and dissemination of scientific research documents, whether they are published or not. The documents may come from teaching and research institutions in France or abroad, or from public or private research centers.
L'archive ouverte pluridisciplinaire HAL, est destinée au dépôt et à la diffusion de documents scientifiques de niveau recherche, publiés ou non, émanant des établissements d'enseignement et de recherche français ou étrangers, des laboratoires publics ou privés. 


\section{The clpP multigenic family in Streptomyces lividans: conditional expression of the clpP3 clpP4 operon is controlled by PopR, a novel transcriptional activator}

\author{
Julie Viala, Georges Rapoport and Philippe Mazodier* \\ Unité de Biochimie Microbienne, CNRS URA 2172, \\ Institut Pasteur, 25 rue du Docteur Roux, 75724 Paris \\ Cedex 15, France.
}

\section{Summary}

The c/pP genes are widespread among living organisms and encode the proteolytic subunit of the Clp ATP-dependent protease. These genes are present in a single copy in most eubacteria. However, five clpP genes were identified in Streptomyces coelicolor. The clpP1 clpP2 operon was studied: mutations affected the growth cycle in various Streptomyces. Here, we report studies of the expression of the clpP3 clpP4 operon in Streptomyces lividans. The clpP3 operon was induced in a clpP1 mutant strain, and the regulation of expression was investigated in detail. The product of the putative regulator gene, downstream from $c / p P 4$, was purified. Gel migration shift assays and DNase I footprinting showed that this protein binds to the clpP3 promoter and recognizes a tandem 6 bp palindromic repeat (TCTGCC-3NGGCAGA). In vivo, this DNA-binding protein, named PopR, acts as an activator of the clpP3 operon. Studies of pop $R$ expression indicate that the regulator is probably controlled at the post-transcriptional level.

\section{Introduction}

Streptomyces are high $\mathrm{G}+\mathrm{C} \%$ Gram-positive soil bacteria that possess an $8 \mathrm{Mb}$ linear chromosome. Sequencing is presently under way and has already revealed many multigene families. Mostly studied for the past 50 years because of its large and diverse production of secondary metabolites, Streptomyces is also a model of bacterial differentiation on account of its complex growth cycle. On solid media, spore germination leads to the growth of basal mycelia, which then differentiate into aerial mycelia that finally septate and differentiate into spores. Two classes of differentiation mutants have been characterized: some present a 'bald' phenotype and are arrested at Accepted 11 August, 2000. *For correspondence. E-mail mazodier@ pasteur.fr; Tel. (+33) 1456888 42; Fax (+33) 145688938 . the basal mycelial state, whereas others are 'white' mutants that are unable to sporulate. The mechanisms regulating cell differentiation in Streptomyces have not been totally elucidated. As energy-dependent proteases are often responsible for the degradation of regulatory proteins (Gottesman and Maurizi, 1992), the role of one of them, the Clp protease, has been investigated in Streptomyces (de Crecy-Lagard et al., 1999). The Clp complex is an ATP-dependent serine protease (Maurizi et al., 1990). In Escherichia coli, it is composed of a tetradecamer of ClpP catalytic subunits, forming a central chamber and associated at one or both ends with a hexamer of ATPase subunits, ClpX or ClpA (ClpC in Gram-positive bacteria) (Kessel et al., 1995; Wang et al., 1997; Grimaud et al., 1998), which confer substrate specificity. Isolated ClpP subunits have been shown to cleave peptides no longer than six amino acids (Woo et al., 1989), and the ATPase subunits serve as chaperones (Wawrzynow et al., 1995). clpP genes are generally present as single copies in eubacteria, but some organisms possess a multigenic $c / p P$ family. For example, two clpP genes are present in Mycobacterium tuberculosis, four in the cyanobacterium Synechococystis and at least five in Streptomyces coelicolor. In S. coelicolor, the five genes are organized as two bicistronic operons and one monocistronic gene, all located at different sites on the chromosome. Although $c / p P$ genes are ubiquitous, they have been analysed in only a few species. Studies in E. coli, Bacillus subtilis, Caulobacter crescentus and Streptomyces lividans implicate ClpP in cell cycle development. Indeed, ClpP protease degrades the stationaryphase sigma factor in E. coli (Schweder et al., 1996), and the $c / p P$ mutant in $B$. subtilis is affected in competence and sporulation (Msadek et al., 1998). In C. crescentus, ClpP is required for normal cell division, growth and viability (Jenal and Fuchs, 1998) and, in S. lividans, the clpP1 c/pP2 mutant has a bald phenotype, i.e. it is unable to form aerial mycelia and thus unable to complete the normal growth cycle (de Crecy-Lagard et al., 1999). In E. coli, the Clp proteolytic complex degrades phage replication proteins (Wojtkowiak et al., 1993; Mhammedi-Alaoui et al., 1994) and proteins tagged by the SsrA system (Gottesman et al., 1998). All other known targets of Clp are regulators: the transcriptional regulator ComK that drives competence development in B. subtilis (Turgay 


\section{A mRNA clpP1}

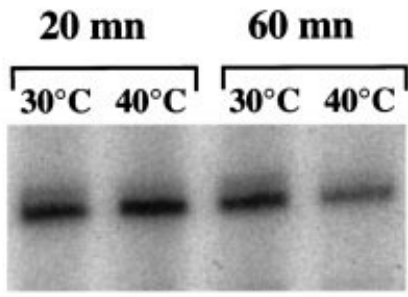

B mRNA clpP3

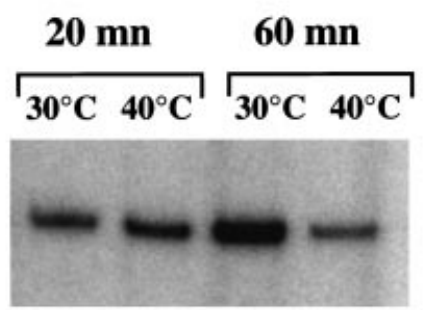

mRNA groES

$60 \mathrm{mn}$

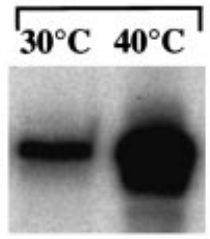

mRNA groES

\section{$60 \mathrm{mn}$}

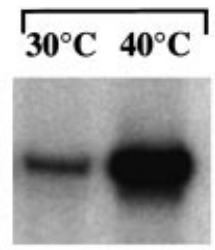

Fig. 1. Studies of the heat shock response of the clpP1 and $c / p P 3$ operons. Analysis by primer extension was done to determine whether $c / p P 1$ and $c / p P 3$ are heat shock genes.

A. For $c / p P 1$, mRNAs were extracted from cultures of wild-type $S$ lividans grown at $30^{\circ} \mathrm{C}$ for $30 \mathrm{~h}$, then kept at that temperature or subjected to heat shock for 20 or $60 \mathrm{~min}$ at $40^{\circ} \mathrm{C}$.

B. For $c / p P 3$, mRNAs were extracted from cultures of the clpP1 mutant subjected to identical heat shock treatments. groES was used as a control to verify heat shock conditions.

et al., 1998) and the multicell cycle processes regulator CtrA in C. crescentus (Jenal and Fuchs, 1998). The Clp protease probably also plays a more general role. Indeed, although $c / p P$ genes are constitutively expressed at a low level, most are strongly induced by many and diverse physiological stresses, such as heat shock, ethanol and puromycin treatments (Gerth et al., 1998; Frees and Ingmer, 1999; Østeras et al., 1999). The stress induction of $c / p P$ genes indicates that they are subject to regulatory systems. To date, only two systems regulating $c / p P$ genes are known: $\sigma^{32}$ the general heat shock sigma factor in $E$. coli (Kroh and Simon, 1990) and the operator-repressor CtsR system identified in B. subtilis (Derré et al., 1999). In $B$. subtilis, under non-stress conditions, the repressor CtsR binds a heptanucleotide repeat (5'-A/GGTCAAANANA/GGTCAAA-3'), which often overlaps the -10 and -35 consensus sequence at the $c / p P$ promoter. Repression is relieved by an unknown mechanism under heat shock conditions. The control by CtsR is found in many low $\mathrm{G}+\mathrm{C}$ Gram-positive bacteria. Neither of these two mechanisms has been reported in Streptomyces, in which three different modes of heat shock regulation have been described. They are all repressor-operator systems: HrcA/CIRCE controls groESL genes (Duchêne et al.,

1994); HspR/HAIR regulates the dnaK operon and $c / p B$ (Bucca et al., 1997; Grandvalet et al., 1997; 1999); and RheA is the repressor of hsp18 in Streptomyces albus (Servant et al. 2000). None of these operators has been identified upstream from Streptomyces clpP genes.

Our experiments gave no evidence that the $c / p P 1 c / p P 2$ operon of $S$. lividans was induced under heat shock conditions. We examined the transcriptional expression of the clpP3 clpP4 operon on the premise that it is implicated in the heat shock response. In the conditions we tested, neither clpP1 nor clpP3 appeared to be heat shock genes. Our findings indicate that expression of the clpP3 clpP4 operon is dependent on the genetic background, as its transcription was induced in a $c / p P 1 c l p P 2$ mutant strain. We also show that this expression is under the control of a specific activator. Thus, we report the first evidence for an interactive regulation between the $c / p P$ multigenic genes.

\section{Results}

Existence of two clpP bicistronic operons in S. coelicolor

Sequencing of the $S$. coelicolor $A 3(2)$ reference strain is under way at the Sanger Center (http://www.sanger.ac.uk/Projects/S_coelicolor/). The nucleotide sequences of two $c / p P$ genes, in addition to the known $c / p P 1$ and $c / p P 2$, have already been determined. These genes, clpP3 and clpP4, are carried by the $5 \mathrm{H} 1$ cosmid, mapping at the right extremity of the chromosome. The putative translation initiation codon of clpP4 is $1 \mathrm{bp}$ downstream from the clpP3 stop codon, which suggests that they are organized as a single operon. The clpP3 ATG initiation codon is preceded by a putative Shine-Dalgarno sequence GGAGC 4 bp upstream. The two genes clpP3 and clpP4 encode predicted proteins of 217 and 200 amino acids with calculated molecular masses of 23.5 and $21.6 \mathrm{kDa}$ respectively. S. coelicolor ClpP1 and ClpP3 are $50.2 \%$ similar, and Clp2 and ClpP4 are $53.7 \%$ similar. All other sequence pairings are less than $41.5 \%$ similar. clpP3 clpP4 may therefore be a paralogous operon of clpP1 clpP2.

In E. coli, Ser-97/His-122/Asp-171 are the catalytic triad of the serine protease ClpP, and Ser and His residues are essential for Clp protease activity (Maurizi et al., 1990; Wang et al., 1997). In Streptomyces, the catalytic residues Ser/His/Asp are perfectly conserved in ClpP1, ClpP2 and ClpP3. However, interestingly, in ClpP4, the His residue is substituted by a Gly residue, and the nearest histidine is 6 amino acids upstream from this position. Whether this has a large effect on ClpP4 catalytic activity, as it would in $E$. coli, remains to be determined. 
clpP1 and clpP3 operons are not heat induced

S. lividans 1326 is very closely related to the $S$. coelicolor A3(2) reference strain and it is easier to manipulate. $S$. lividans 1326 was therefore used for the following experiments.

To determine the promoter region of the clpP3 clpP4 operon, primer extension analysis was performed. No transcripts were revealed using mRNAs extracted from the $S$. lividans wild-type strain. clpP genes have been described as heat shock genes in many organisms, so primer extensions were carried out on mRNAs extracted from S. lividans cultures after heat shock at $40^{\circ} \mathrm{C}$ for 20 and $60 \mathrm{~min}$. No heat-induced signal could be detected (not shown). Absence of heat induction was also observed for the clpP1 clpP2 operon (Fig. 1A). These results suggest that, at least in the standard conditions assayed, which are known to induce many Streptomyces heat shock proteins (HSPs) (Puglia et al., 1995), clpP1 or clpP3 operons are not heat shock induced.

Conditional expression of the clpP3 clpP4 operon from S. lividans and determination of the transcriptional start site

The S. lividans clpP1 mutant is unstable, and the bald phenotype could be lost on certain media after subculturing, although the clpP1 gene is still disrupted (de CrecyLagard et al., 1999). This indicates that either compensatory mutations appear or usually silent genes are activated. Our results indicated a silencing of the clpP3 operon in the wild-type strain, and the clpP3 operon appears to be a paralogue of the clpP1 operon. Thus, the reversion of the bald phenotype of the $c / p P 1$ mutant may result from the expression of the clpP3 operon being turned on. mRNAs were extracted from the wild-type strain and from two clpP1 mutant strains, one bald and one showing a partial revertant phenotype, and used for primer extension analysis. No signal was detected for the wild-type RNA, and strong signals were observed for RNA from both clpP1 mutants (Fig. 2). mRNA from the glucose kinase gene ( $g / k)$ was used as a quantitative control. Expression of $c / p P 3 c / p P 4$ was demonstrated consistently only in the clpP1 mutant; this does not preclude the possibility of a low-level transient expression in a wild-type in conditions that were not established. Indeed, it is well referenced that there are considerable variations in protein synthesis throughout the cell cycle in Streptomyces. In view of these results, we reanalysed clpP3 clpP4 transcripts in heat shock conditions in a clpP1 mutant. No heat induction was observed (Fig. 1B).

Maintenance of a bald phenotype in the clpP1 mutant strain expressing clpP3 and clpP4 indicates that ClpP3 and ClpP4 are not fully isofunctional to ClpP1 and ClpP2

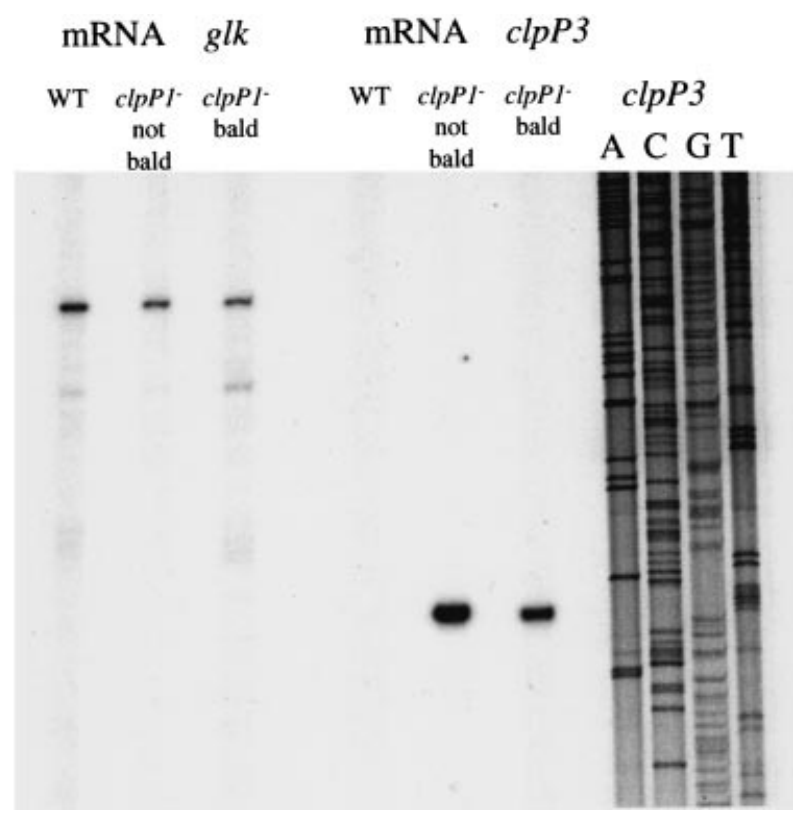

Fig. 2. Expression analysis and mapping of the $5^{\prime}$ end of the $c / p P 3$ mRNA. In order to determine the transcription start site and to evaluate the level of $c / p P 3$ expression, primer extensions were performed on the clpP3 mRNA. mRNAs were extracted from cultures of the wild-type strain (WT), a clpP1 mutant presenting the bald phenotype (c/pP1 ${ }^{-}$bald) and a $c / p P 1$ mutant strain showing a partial revertant phenotype ( $c / p P 1^{-}$not bald). A control was carried out on the glucose kinase ( $g / k)$ mRNA extracted from same cultures; upper bands correspond to the predicted signal. Lanes A, $\mathrm{C}, \mathrm{G}$ and $\mathrm{T}$ show the product of the dideoxy sequencing realized on pJV37 with the same primer used for the primer extension of the clpP3 mRNA.

but suggests some specificity of action of the various CIpP paralogues.

A single transcription start site was localized $83 \mathrm{bp}$ upstream from the $c / p P 3$ translation initiation codon. None of the Streptomyces promoter consensus sequences described by Bourn and Babb (1995) could be identified in this region.

\section{Overexpression and purification of PopR, a putative DNA- binding protein}

Cosmid $5 \mathrm{H} 1$ has an open reading frame (ORF) downstream from the clpP4 gene in the opposite orientation (Fig. 3). This ORF encodes a putative DNA-binding protein of 157 amino acids with a calculated molecular mass of $16.7 \mathrm{kDa}$. A five-amino-acid segment (RPQAT) is repeated four times in the $\mathrm{N}$-terminal region, and a helixturn-helix $(\mathrm{HTH})$ motif is present between the 77th and 97th amino acid residues of the protein. The putative protein shows no similarity to proteins in databanks except for three other putative DNA-binding proteins, one in $S$. coelicolor and two in another Actinomycete, 


\section{0 bp}

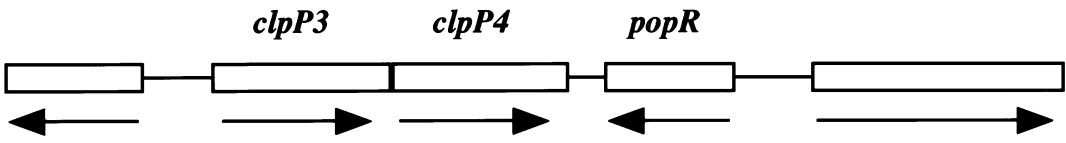

Fig. 3. Genetic organization of the $c / p P 3$ operon region. Arrows indicate the direction of gene transcription.
Mycobacterium tuberculosis. However, most of the sequence identities are in the HTH motif.

We investigated whether this putative DNA-binding protein, called PopR for clpP 3 operon regulator, is a regulator of the clpP3 clpP4 operon. To assay the DNAbinding activity of PopR, it was overproduced in $E$. coli and purified as follows (Fig. 4). The popR gene from $S$. lividans was amplified by PCR, and the product was inserted into the vector pET28. The sequence of the gene was determined: the $S$. lividans PopR is nine amino acid residues shorter than its $S$. coelicolor counterpart because of the absence of one RPQAT motif and a stretch of four amino acid residues in the C-terminal region. The construction, pEt28popR, was introduced into E. coli for IPTG-inducible T7-driven overproduction of a recombinant PopR protein carrying a His-tag at its $\mathrm{N}$ terminus. The recombinant PopR was purified on column. The His-tag was removed from the PopR recombinant protein by treatment with thrombin, a site-specific protease.

\section{PopR binds directly to the clpP3 promoter region}

Gel mobility shift DNA-binding assays were used to test

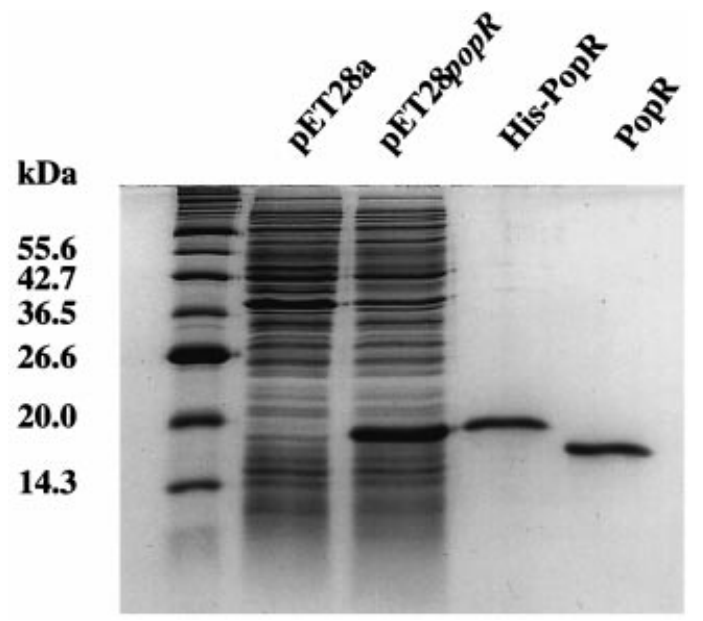

Fig. 4. Overproduction and purification of PopR. Crude extracts were prepared from cultures of $E$. coli BL21 $\lambda D E 3$ carrying pET28a (lane pET28a) or pET28popR (lane pET28popR). These samples, as for His-tag PopR recombinant protein purified on Ni-NTA column (lane His-PopR) and purified PopR after thrombin treatment (lane PopR), were loaded on 15\% Tris-glycine SDS-PAGE. Molecular weight standards (Biolabs) are on the left. the DNA-binding activity of PopR with the clpP3 promoter region: a fragment of $330 \mathrm{bp}$ corresponding to positions -247 to +82 relative to the transcription start site of $c / p P 3$ was obtained by PCR amplification. Similar experiments were carried out on a $310 \mathrm{bp}$ PCR-amplified fragment corresponding to the upstream region of $p o p R$ to test for possible autoregulation of the gene.

The prepared radiolabelled fragments were incubated with increasing amounts of purified PopR. Complete displacement was observed when the clpP3 promoter region was incubated with the highest quantity of PopR tested (200 ng); no displacement was observed when the popR upstream region was incubated with the same quantity of protein (Fig. 5). Thus, PopR bound specifically to the clpP3 promoter region but was not subject to autoregulation.

\section{PopR recognizes a double-tandem 6 bp palindrome}

The operator site in the $c / p P 3$ promoter region recognized by PopR was determined by DNase I footprint assays. A radiolabelled fragment of $360 \mathrm{bp}$ corresponding to positions -160 to +196 relative to the transcription start site was amplified by PCR and treated with DNase I. The PopR protein protects one region extending from positions -75 to -35 relative to the transcription start site (Fig. 6). The following double-inverted repeat, extending from positions -70 to -41 (Fig. 7), was identified in the protected region: TCTGCC-3N-GGCGAA TCTGCC-3NGGCAGA.

Numerous $\sigma^{70}$ promoters in $E$. coli subject to activation contain an activator site close to the -40 position (Gralla, 1991). Possibly, the DNA-binding protein PopR, which binds to the clpP3 promoter, is an activator that allows RNA polymerase recruitment.

PopR activates expression of the clpP3 operon in S. lividans

To examine whether PopR is an activator, it was overexpressed in various $S$. lividans strains, and $c / p P 3$ clpP4 operon expression was assessed by primer extension.

A $780 \mathrm{bp}$ fragment corresponding to $p o p R$ and its promoter region was amplified by $\mathrm{PCR}$ and inserted into pUWL219. The resulting construction, pJV100, allowed 

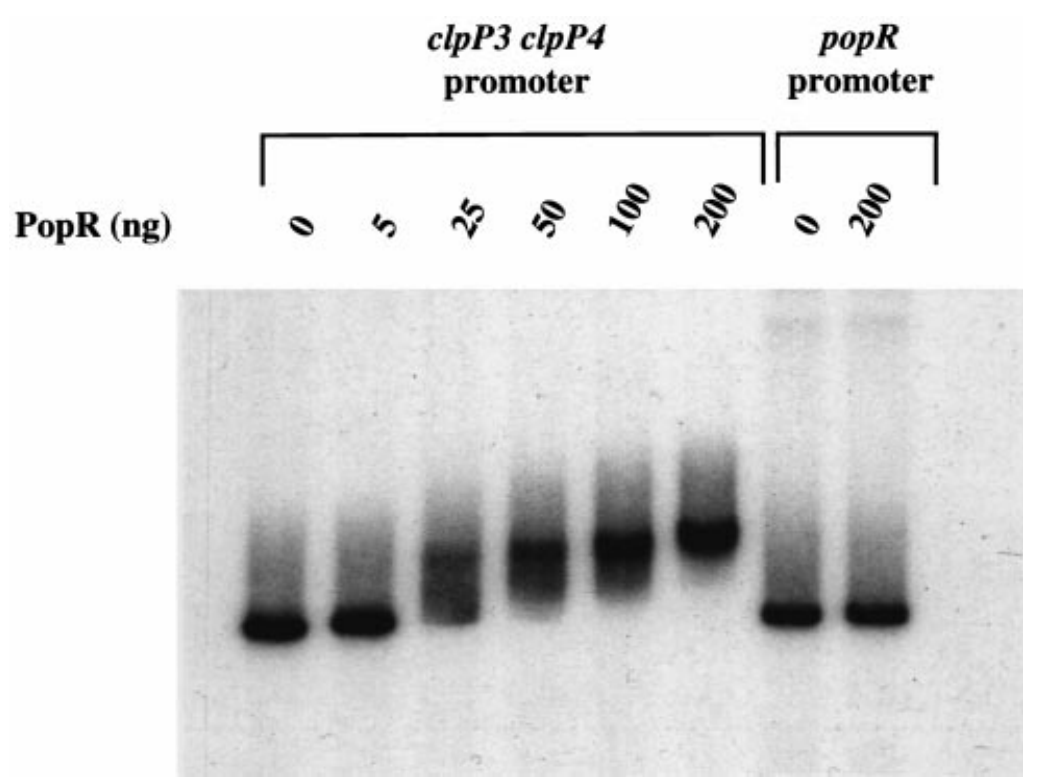

Fig. 5. Specific binding of PopR to the $c / p P 3$ promoter region revealed by the gel shift experiment. The 330 bp DNA fragment probe (10 000 c.p.m.) corresponding to the $c / p P 3$ promoter region was incubated with increasing amounts of purified PopR (0$200 \mathrm{ng})$. A 310 bp DNA fragment probe (10 000 c.p.m.) corresponding to the popR upstream region was incubated with 0 and 200 ng of purified PopR.
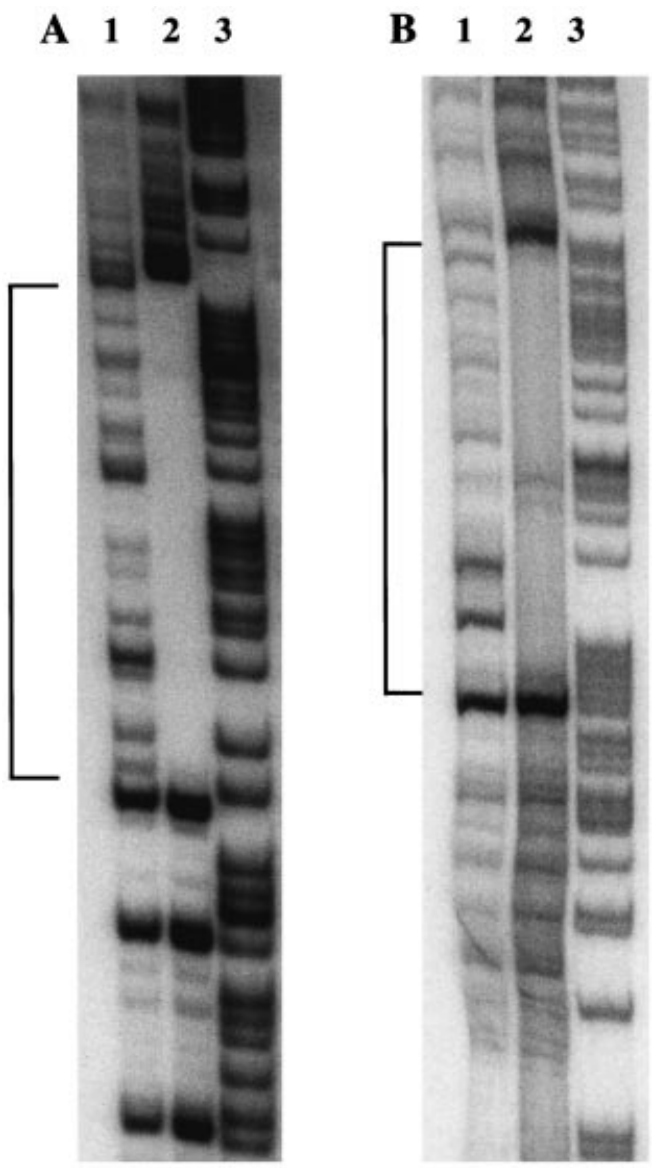

Fig. 6. Determination of the operator site recognized by PopR. Approximately 50000 c.p.m. of labelled template strand (A) and non-template strand $(\mathrm{B})$ of the $c / p P 3$ promoter region were submitted to DNase I treatment without (lane 1) or with previous incubation with $1 \mu \mathrm{g}$ of purified PopR (lane 2). G+A Maxam and Gilbert reactions of the appropriate labelled DNA fragments were also loaded (lane 3). Brackets indicate the region protected by PopR. the expression of popR under its own promoter on a multicopy vector. pJV100 was introduced into S. lividans 1326 wild-type and c/pP1 mutant strains by conjugation from E. coli S17.1. Conjugants were selected on thiostrepton. mRNAs were extracted from cultures of the strains harbouring or not harbouring pJV100. $c / p P 3$ transcription was analysed by primer extension, and $g / k$ mRNA was used for RNA quantification controls. A strong signal was detected with mRNAs extracted from the $c / p P 1$ mutant strain overexpressing $p o p R$. The signal was weaker with mRNAs extracted from the clpP1 mutant. Interestingly, overexpression of $p o p R$ in a wildtype strain led to the expression of $c / p P 3$ (Fig. 8). We concluded that PopR is the activator of the clpP3 clpP4 operon.

\section{Attempts to obtain a clpP1 popR double mutant}

We attempted to construct a strain with a $c / p P 1$ popR double mutation. However, despite considerable effort, no such mutant was obtained. PopR being the activator of $c / p P 3$, one predicted characteristic of this construction would be the absence of clpP3 clpP4 transcription. To understand the impossibility of achieving this construction, we tried to obtain a clpP1 clpP3 double mutant by targeting the structural genes directly. Whereas simple clpP1 and clpP3 mutants were readily selected, the double mutant was not obtained. The phenotype of the clpP3 mutant is different from the phenotype of $c / p P 1$; whereas $c / p P 1$ is bald, the clpP3 mutant is not affected in its cell cycle, yet it presents an increased production of a blue pigment (actinorhodin). 


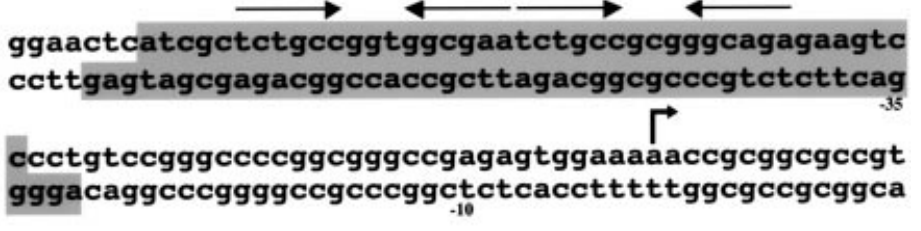

tcggccgcacacccegcaccacctccgcacgaccggccacgaccggcc agccggcgtgtggggcgtggtggaggcgtgctggceggtgctggcegg

acgactcgacaagras

tgecgatgtctccattcaccgccggcccegce

tgctgagctgttcctcgcggctacagaggtaagtggcggccggggcgg $\begin{array}{lllllllll}M & \mathbf{S} & \mathbf{P} & \mathbf{F} & \mathbf{T} & \mathbf{A} & \mathbf{G} & \mathbf{P} & \mathbf{A}\end{array}$
Fig. 7. Nucleotide sequence of the $c / p P 3$ promoter region and the deduced amino acid sequence of the beginning of the gene. The putative ribosome binding site is boxed, the +1 position start is indicated by $r$ and nucleotides -10 and -35 are indicated. The Dnase I protected areas are shaded, and the PopR double-inverted repeat recognition target is indicated by inverted arrows in the sequence.

\section{Transcriptional expression of popR}

To determine whether $c / p P 3$ activation by PopR in a $c / p P 1$ mutant resulted from popR enhanced expression, the rate of transcription of $p o p R$ was evaluated. Primer extension analysis was carried out using two different primers. A unique transcription start point was identified. It is preceded by two hexamers (TAGGCT and TTACGG) at positions -10 and -35 . The -10 sequence is in good agreement with the consensus motif recognized by the vegetative HrdB sigma factor (Strohl, 1992), whereas the -35 sequence presents a lower similarity. This transcription start site is located at the initiation codon. Such leaderless mRNAs have been described for other Streptomyces genes (Jones et al., 1992). The signals were easily detectable with mRNAs from strains harbouring pJV100, but were very faint with mRNAs from strains possessing only the chromosomal copy of popR. The

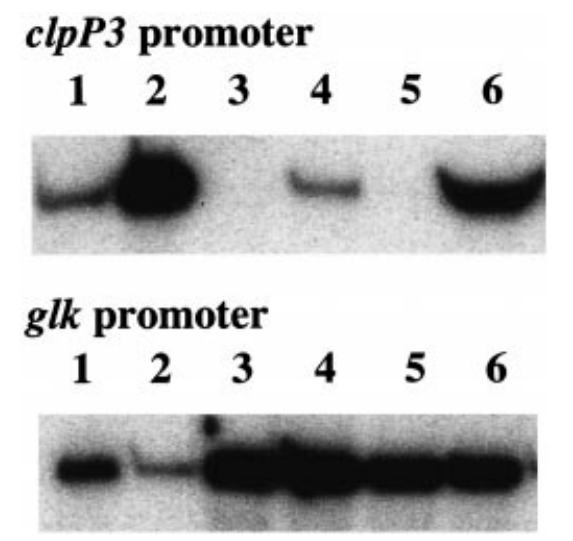

Fig. 8. $c / p P 3$ PopR-dependent expression. Primer extensions were realized on $c / p P 3$ mRNA. mRNAs were extracted from the $c / p P 1$

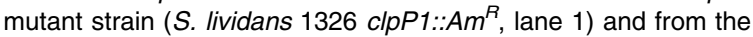
clpP1 mutant strain overexpressing popR present in multicopy [S. lividans 1326 clpP1::Am ${ }^{R}$ (pJV100), lane 2]. mRNAs were also extracted from the wild-type strain carrying the empty multicopy vector at $22 \mathrm{~h}$ or $32 \mathrm{~h}$ of growth culture [S. lividans 1326 (pUWL219), lanes 3 and 5 respectively] and from wild-type strain overexpressing popR at $22 \mathrm{~h}$ or $32 \mathrm{~h}$ of growth culture [S. lividans 1326 (pJV100), lanes 4 and 6 respectively]. intensity of the signals was similar for the wild-type and clpP1 mutant strains (Fig. 9). This indicates that ClpP1 has no direct effect on the transcriptional regulation of popR. Accordingly, accumulation of $c / p P 3$ transcripts in the clpP1 mutant suggests a post-transcriptional regulation of the activator.

\section{Discussion}

This is the first report describing an interactive regulation among the clpP multigenes in Streptomyces. In the present study, we have shown that $c / p P 3 \quad c / p P 4$ operon expression is induced when the $c / p P 1 c / p P 2$ operon is not functional. This induction involves an activator, PopR, encoded by a gene downstream from $c / p P 4$ and in the opposite orientation. The PopR protein directly binds a

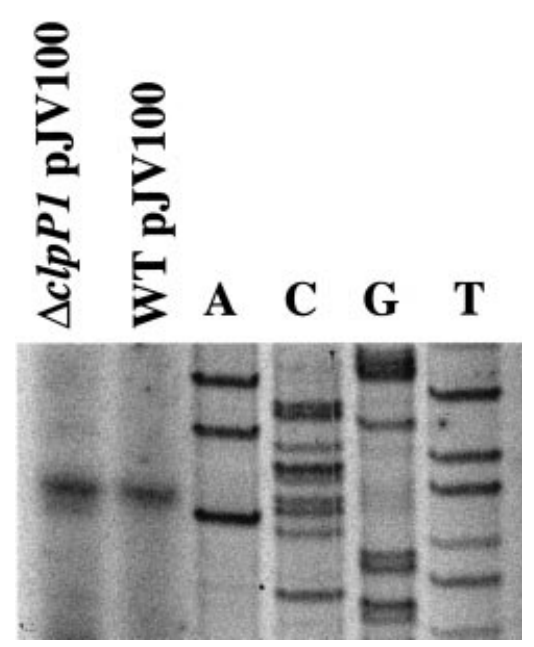

Fig. 9. Expression analysis and mapping of the $5^{\prime}$ end of the popR mRNA. In order to determine the transcription start site and to evaluate the eventual effect of the clpP1 mutant genetic background on $p o p R$ transcription, primer extensions were performed on the popR mRNA extracted from cultures of the $c / p P 1$ mutant overexpressing popR (clpP1- pJV100) or from the wild-type strain overexpressing popR (WT pJV100). Lanes A, C, G and T show the product of the dideoxy sequencing realized on pJV100 with the same primer as that used for the primer extension. 
double-tandem repeat of a 6 bp palindromic sequence and activates the transcription of the $c / p P 3 c / p P 4$ genes. $\mathrm{PopR}$ is the first activator involved in $c / p P$ regulation described in bacteria. In the sequence data available for the $S$. coelicolor genome, the complete operator site recognized by PopR is only found upstream from $c / p P 3$, suggesting that $P o p R$ is not a pleiotropic regulator. None of the promoter consensus classes defined by Bourn and Babb (1995) could be identified in the clpP3 promoter region other than a CNGNNA hexamer. This motif begins at position -13 upstream from the transcription start site, a position rarely used according to the data of Bourn and Babb (1995). This motif is usually associated with another sequence TANNNT or CANNAT, not present in the $c / p P 3$ promoter, probably indicating weak RNA polymerase promoter-binding activity. PopR binding at position -40 presumably recruits the RNA polymerase.

Surprisingly, considering reports on the heat shock induction of $c / p P$ in many eubacteria, we have not observed heat shock induction either for $c / p P 1$ or for $c / p P 3$ in standard conditions. However, the heat shock modulon presents several peculiarities in Streptomyces. Puglia et al. (1995) have shown that there is a lot of variation in the patterns of kinetics of heat and developmentally induced synthesis of the different heat shock proteins in $S$. coelicolor, and we have not achieved such an exhaustive study of this phenomenon on clpP genes.

The $c / p P 3$ operon and the popR gene map at the right extremity of the $S$. coelicolor linear chromosome within an unstable region. Indeed, Streptomyces chromosomal ends are subject to substantial genetic instability: large-scale deletions and DNA sequence amplifications may occur with a frequency $>10^{-3}$ (Fischer et al., 1998; Volff and Altenbuchner, 1998). Chromosomal ends are proposed to contain nonessential or conditionally essential genes (Redenbach et al., 1996). Conditional expression of the clpP3 operon in a clpP1 mutant is in agreement with this hypothesis. Furthermore, whereas simple $c / p P 1$ and clpP3 mutants were readily selected, the double mutant was not obtained. This might suggest that at least one $c / p P$ bicistronic operon is required for viability. Such a critical role of $\mathrm{ClpP}$ in cell viability has been reported in C. crescentus (Jenal and Fuchs, 1998). Interestingly, the two $c / p P$ operons functions overlap only partially. Indeed, $c / p P 3$ and $c / p P 4$ do not fully complement the phenotype of the $c / p P 1 \quad c / p P 2$ mutant strain: the bald phenotype persists even after induction of the clpP3 operon. Furthermore, the clpP3 mutant does not present the bald phenotype. These results point to different specific roles for the various ClpP proteins. Four distinct ATPases belonging to the HSP100 family have been identified in the $S$. coelicolor chromosome: two encoded by $c l p C$ genes, one by $c l p X$ and one by $C / p B$ (no interaction is known with ClpP for the last). There are also five $c / p P$ genes in this species. It is not known whether there is a specificity of interaction between the different HSP100 Clp proteins and the $\mathrm{ClpP}$ protease. If there is, ClpP1 and ClpP2 could have targets other than those of $\mathrm{ClpP} 3$ and $\mathrm{ClpP} 4$ because substrate specificity is conferred by the HSP100. This could explain why $c / p P 3$ and $c / p P 4$ do not fully complement the phenotype linked to the $c / p P 1 \quad c / p P 2$ mutations. In view of the substitution of His by Gly in the catalytic triad, the activity of $\mathrm{ClpP} 4$ should be examined further. The histidine residue nearest to the position predicted by the alignments is six amino acid residues upstream. It is possible that $\mathrm{ClpP} 4$ is inactive. Another organism, the cyanobacterium Synechocystis, contains four clpP genes, one of which, clpP4, lacks all three amino acids residues of the catalytic triad. Porankiewicz et al. (1999) proposed that this protein is not a true ClpP but a ClpR, a new uncharacterized class of protease.

PopR is the centre of the regulation system and, thus, the expression of clpP3 clpP4 is dependent on its cellular level and activity. As the expression of popR is similar in the clpP1 mutant and wild-type strains, the control of the activator is presumably post-transcriptional. ClpP1 and ClpP2 are involved in the stability or activity of PopR, and PopR might be a target for the Clp protease. No specific motif has been characterized in the substrates of the Clp proteolytic complex; however, in $E$. coli, the $\mathrm{N}$-terminal and $\mathrm{C}$-terminal peptide sequences are important for signal recognition. This is illustrated by the $\mathrm{N}$-end rule and by the SsrA-tagging system. The $\mathrm{N}$ end rule is that proteins carrying certain amino acids at the $\mathrm{N}$-terminus are highly unstable and ClpAP is implicated in their degradation (Tobias et al., 1991). The SsrA-tagging system, widespread among eubacteria, adds a C-terminal peptide tag to proteins as a degradation signal (Tu et al., 1995; Keiler et al., 1996; Roche and Sauer, 1999). Among bacteria, there is a quasi-absolute conservation of the Leu-Ala-Ala motif at the C-terminal extremity of the peptide tag. It has been shown that these two Ala are crucial for the degradation by ClpAP or ClpXP proteases (Gottesman et al., 1998). The fact that the peptide sequence of PopR ends with Leu-Ala-Ala further sustains the hypothesis that ClpP1 and ClpP2 might degrade this protein. This question will be addressed in future work.

Recently, a fifth $c / p P$ gene from $S$. coelicolor was sequenced (http://www.sanger.ac.uk/Projects/S_coelicolor/). Nothing is yet known about it. The role of this new gene needs investigation, and its involvement in the general regulatory network of the other clpP genes remains to be elucidated. 


\section{Experimental procedures}

\section{Bacterial strains and media}

S. lividans strain 1326 was obtained from the John Innes Culture Collection, and $S$. lividans $1326 \mathrm{clpP1}: \mathrm{Am}^{\mathrm{R}}$ was constructed in this laboratory (de Crecy-Lagard et al., 1999). YEME medium was used for liquid growth (Hopwood et al., 1985). NE medium (Murakami et al., 1989) and R5 (Hopwood et al., 1985) were used for Streptomyces growth on plates. The antibiotics apramycin and thiostrepton were added to final concentrations of $25 \mu \mathrm{g} \mathrm{ml}^{-1}$ to solid medium and to 20 and $10 \mu \mathrm{g} \mathrm{ml}^{-1}$, respectively, to liquid medium.

E. coli TG1 (Gibson, 1984) was used as the general cloning host, E. coli S17-1 (Simon et al., 1983) was used as the conjugative host and $E$. coli BL21 $\lambda$ DE3 for protein production and purification (Studier and Moffatt, 1986). E. coli strains were grown in LB medium. The antibiotics ampicillin and kanamycin were added to final concentrations of 100 and $25 \mathrm{\mu g} \mathrm{ml}^{-1}$ respectively.

\section{DNA manipulation, transformation and conjugation procedures}

Plasmid DNA was extracted from E. coli using a Qiagen kit. DNA fragments were purified from agarose gels with Ultrafree-DA (Amicon-Millipore). Restriction enzymes were used as recommended by the manufacturers. DNA fragments were amplified by the PCR technique (Mullis and Faloona, 1987; Saiki et al., 1988). DNA sequences were determined by the dideoxy chain termination method (Sanger et al., 1977) using a modified T7 DNA polymerase (Pharmacia). Standard $\mathrm{CaCl}_{2}$ (Cohen et al., 1972) or electroporation procedures were used for $E$. coli transformation.

Streptomyces DNA was prepared as described by Hopwood et al. (1985). Intergenic conjugations from E. coli S17-1 to $S$. lividans 1326 or $S$. lividans 1326 clpP1::Am ${ }^{\mathrm{R}}$ were performed as described previously (Mazodier et al., 1989).

\section{Plasmids and plasmid constructions}

The E. coli vectors used were pUC19 (Yanisch-Perron et al., 1985) for cloning and pET28a (Novagen) for overproduction and purification of proteins. The Streptomyces/E. coli shuttle vectors used were pUWL219 (Wehmeier, 1995) and pGM160 $\Delta$ made by J. L. Pernodet and obtained by deleting the Apra $^{\mathrm{R}} 1610$ bp HindllI fragment from the thermosensitive vector pGM160 (Muth et al., 1989).

The transcription start site of $c / p P 3$ was localized by a sequencing reaction with the oligonucleotide used in primer extension on plasmid pJV37. pJV37 consists of the $1800 \mathrm{bp}$ fragment including the $S$. lividans 1326 promoter region and the clpP3 clpP4 operon obtained by PCR amplification with primers Ju 40 (see Table 1) and Ju 43, inserted between the EcoRI and BamHI sites of pUC19. To overproduce PopR, pET28popR was constructed by inserting the 475 bp fragment including the $S$. lividans 1326 popR coding sequence obtained by PCR amplification with primers Reg1 and Reg3 between the Ndel and BamHI sites of pET28a. This construction led to an $\mathrm{N}$-terminal translational fusion between a peptide of 20 amino acids containing six $\mathrm{His}$ residues and
Table 1. Primers used in this study.

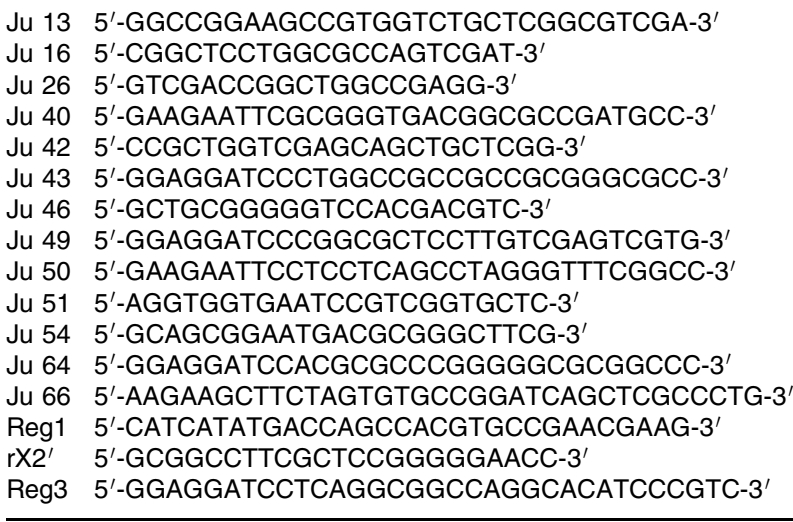

PopR under the control of the strong inducible T7 promoter. To overexpress popR in Streptomyces, pJV100 was constructed. pJV100 is the $780 \mathrm{bp}$ fragment including the promoter region and $p o p R$ gene obtained by PCR amplification with primers Ju46 and Reg3 inserted into the blunted BamHI site of pUWL219. In this construction, popR is under the control of its own promoter on a multicopy vector. An oriT fragment was inserted into the Pstl site to allow conjugation from $E$. coli to $S$. lividans.

The clpP3 gene was disrupted as follows. clpP3 was PCR amplified using oligonucleotides Ju40 and Ju43 and cloned into pUC19. The resulting plasmid was digested with Sfi, blunt ended by Vent polymerase treatment and ligated with

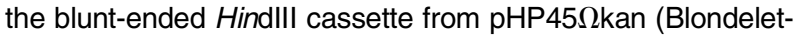
Rouault et al., 1997) containing the neomycin-kanamycin resistance gene (kan). The EcoRI-BamHI clpP3::kan bluntended fragment was cloned into pGM160s at the bluntended BamHI site to give plasmid pJV42. Treatment and selection of $c / p P 3$ mutants by double recombination was as described by de Crecy-Lagard et al. (1999).

The strategy for disrupting $p o p R$ was the following. A $300 \mathrm{bp}$ popR internal fragment was PCR amplified using oligonucleotides Ju64 and Ju66. It was BamHI-HindIIIdigested and inserted at the corresponding sites in pGM160 $\Delta$ to give pJV108. Precultures of the S. lividans clpP1 mutant transformed with pJV108 were agitated for 3 days at $40^{\circ} \mathrm{C}$ and plated to give isolated colonies on $\mathrm{NE}$ supplemented with thiostrepton.

\section{Heat shock induction in Streptomyces}

Cultures of S. lividans 1326 wild type or clpP1 mutant grown for 24 or $30 \mathrm{~h}$ in YEME were subjected to 20 or $60 \mathrm{~min}$ treatment at $40^{\circ} \mathrm{C}$ or kept at $30^{\circ} \mathrm{C}$. mRNA was extracted from these cultures and analysed.

\section{RNA extraction}

A volume of $10-15 \mathrm{ml}$ of $S$. lividans 1326 or S. lividans 1326 clpP1::Am ${ }^{\mathrm{R}}$ culture was pelleted. Cells were resuspended in $0.5 \mathrm{ml}$ of cold deionized water and added to $0.5 \mathrm{~g}$ of glass beads (212-300 $\mu \mathrm{m}$; Sigma), $0.4 \mathrm{ml}$ of $4 \%$ Bentone (Rheox) 
and $0.5 \mathrm{ml}$ of phenol-chloroform-isoamyl alcohol, $\mathrm{pH} 8.0$ (Amresco). The cells were disrupted in a Fastprep disintegrator (Savant; Bio101) for $30 \mathrm{~s}$ at $4^{\circ} \mathrm{C}$. After centrifugation for $8 \mathrm{~min}$ at $4^{\circ} \mathrm{C}$ and 14000 r.p.m., supernatants were collected, treated with phenol-chloroform $(1: 1, \mathrm{v} / \mathrm{v})$ and then with chloroform-isoamyl alcohol (24:1, v/v). RNA was precipitated at $-20^{\circ} \mathrm{C}$ with $850 \mu \mathrm{l}$ of isopropanol in the presence of $0.2 \mathrm{M} \mathrm{NaCl}$ and resuspended in $20 \mu \mathrm{l}$ of cold deionized water. RNA concentrations were determined by measuring absorbance at $260 \mathrm{~nm}$.

\section{Primer extension experiments}

Primer extensions were performed with purified oligodeoxynucleotides Ju 26 and Ju 42 (see Table 1) on the clpP1 mRNA and clpP3 mRNA, respectively, and with purified oligodeoxynucleotides Ju 13 and Ju 16 on the groES mRNA and $g l k$ mRNA respectively. Primer extensions were also performed on popR mRNA with oligonucleotides Ju 54 and rX2'. The synthetic primer $(10 \mathrm{pmol})$ was $5^{\prime}$ end labelled with $5 \mu$ l of $\left[\gamma^{-}{ }^{32} \mathrm{P}\right]-A T P\left(3000 \mathrm{Ci} \mathrm{mmol}^{-1}\right)$ using T4 polynucleotide kinase. RNA $(40 \mu \mathrm{g})$ and $1 \mathrm{pmol}$ of labelled oligonucleotide were annealed in a final volume of $17 \mu \mathrm{l}$ of $1 \times \mathrm{AMV}$ reverse transcriptase buffer (Boehringer Mannheim) for $3 \mathrm{~min}$ at $65^{\circ} \mathrm{C}$. The mixture was then frozen for $1 \mathrm{~min}$ in dry ice and thawed in ice. Twenty-five units of AMV reverse transcriptase (Boehringer Mannheim) and $2 \mu \mathrm{l}$ of $20 \mathrm{mM}$ dNTP were added. After $30 \mathrm{~min}$ of incubation at $42^{\circ} \mathrm{C}, 1 \mu \mathrm{g}$ of RNase was added, and the mixture was incubated for $10 \mathrm{~min}$ at $37^{\circ} \mathrm{C}$. The reactions were stopped by adding $5 \mu \mathrm{l}$ of a loading solution containing $97.5 \%$ deionized formamide, $10 \mathrm{mM}$ EDTA, $0.3 \%$ xylene cyanol and 3.3\% bromophenol blue. Samples were loaded on $6 \%$ acrylamide urea sequencing gels.

\section{Overproduction and purification of PopR}

pET28popR containing the translational fusion $6 \mathrm{His} / p o p R$ under the control of the T7 promoter was introduced into the E. coli BL21 $\lambda \mathrm{DE} 3$ strain in which the T7 RNA polymerase gene is under the control of the IPTG-inducible lacUV5 promoter. The resulting strain was grown at $37^{\circ} \mathrm{C}$ in $\mathrm{LB}$ medium containing $25 \mu \mathrm{g} \mathrm{ml}^{-1}$ kanamycin until the culture reached an $\mathrm{OD}_{600}$ of 0.9 ; then IPTG was added to a final concentration of $1 \mathrm{mM}$. Incubation was pursued for $6 \mathrm{~h}$ at room temperature. Cells were centrifuged for $10 \mathrm{~min}$ at 5000 r.p.m., and the pellet was resuspended in 1/50th of the culture volume of buffer $1\left(50 \mathrm{mM} \mathrm{NaPO}_{4}, \mathrm{pH} 8,300 \mathrm{mM}\right.$ $\mathrm{NaCl}, 20 \mathrm{mM}$ imidazole). Cells were disrupted by sonication, and cell debris was pelleted by centrifugation for $20 \mathrm{~min}$ at 12000 r.p.m. The E. coli crude extract was loaded on a $100 \mu \mathrm{l}$ Ni-NTA agarose (Qiagen) column previously equilibrated with buffer 1 . The column was washed abundantly with buffer $2\left(50 \mathrm{mM} \mathrm{NaPO}_{4}, \mathrm{pH} \mathrm{6,300} \mathrm{mM} \mathrm{NaCl}, 30 \mathrm{mM}\right.$ imidazole), and the PopR protein was eluted with an imidazole gradient $(30-500 \mathrm{mM})$. The eluted product was dialysed overnight at $4^{\circ} \mathrm{C}$ in $50 \%$ glycerol, $50 \mathrm{mM} \mathrm{NaPO}_{4}$, $\mathrm{pH} 6.5$, and $300 \mathrm{mM} \mathrm{NaCl}$. The 20 residues brought by the translational fusion were removed by thrombin digestion, which cleaves a specific site just upstream from the popR coding sequence, and the thrombin was eliminated as recommended by the manufacturer (Thrombin Cleavage Capture Kit; Novagen).

Total or purified protein extracts were resolved by SDS-15\% PAGE according to the method of Laemmli (1970). The protein concentration was determined by the method of Bradford (1976).

\section{Gel mobility shift DNA-binding assays}

A 330 bp EcoRI-BamHI DNA fragment PCR amplified with primers Ju 40 and Ju 49 (see Table 1), corresponding to the clpP3 promoter region, and a $310 \mathrm{bp}$ EcoRI DNA fragment PCR amplified with primers Ju 46 and Ju 50, corresponding to the popR upstream region, were end labelled with $\left[\alpha-{ }^{32} \mathrm{P}\right]-$ dATP using the Klenow fragment of DNA polymerase I (Gibco-BRL). DNA fragments were then purified using the Qiaquick PCR purification kit (Qiagen). Radiolabelled fragments (10 000 c.p.m.) and various quantities of PopR, from 0 to $200 \mathrm{ng}$, were incubated for $15 \mathrm{~min}$ at $30^{\circ} \mathrm{C}$ in a $10 \mu \mathrm{l}$ reaction containing $1 \mu \mathrm{g}$ of calf thymus (non-specific competitor) DNA and the reaction buffer $\left[25 \mathrm{mM} \mathrm{NaPO}_{4}, \mathrm{pH} 7\right.$, $150 \mathrm{mM} \mathrm{NaCl}, 0.1 \mathrm{mM}$ EDTA, $2 \mathrm{mM} \mathrm{MgSO}_{4}, 1 \mathrm{mM}$ dithiothreitol (DTT), $10 \%$ glycerol]. Samples were then loaded on a $6 \%$ polyacrylamide gel $(50 \mathrm{mM}$ Tris, $400 \mathrm{mM}$ glycine, $1.73 \mathrm{mM}$ EDTA), and electrophoresis was performed under $100 \mathrm{~V}$ tension for $1 \mathrm{~h}$.

\section{DNase / footprint}

Purified synthetic primers Ju 42 and Ju 51 were end labelled using $\left[\gamma_{-}{ }^{32} \mathrm{P}\right]-\mathrm{dATP}$ and the T4 polynucleotide kinase. The clpP3 promoter region was PCR amplified using end-labelled primers Ju 42 and Ju 51 (20 pmol) and Pfu polymerase (Stratagene). Fragments were then purified using the Qiaquick PCR purification Kkit (Qiagen). In a final volume of $50 \mu \mathrm{l}$, PopR $(1 \mu \mathrm{g})$ and end-labelled fragments (50 000 c.p.m.) were incubated for $20 \mathrm{~min}$ at room temperature in the presence of $5 \mu \mathrm{g}$ of calf thymus DNA, $1 \mu \mathrm{g}$ of BSA and $1 \times$ reaction buffer $(10 \mathrm{mM}$ Tris- $\mathrm{HCl}, \mathrm{pH} 7.5,50 \mathrm{mM} \mathrm{NaCl}$, $2.5 \mathrm{mM} \mathrm{MgCl} 2,0.5 \mathrm{mM}$ DTT, $4 \%$ glycerol). Then, $5 \mu \mathrm{l}$ of $10 \mathrm{mM} \mathrm{MgCl}_{2}$ and $5 \mathrm{mM} \mathrm{CaCl}_{2}$ were added, followed by $5 \mathrm{ng}$ of DNase I and the mixture was incubated for $1 \mathrm{~min}$. The reaction was stopped with $140 \mu \mathrm{l}$ of stop buffer $(0.4 \mathrm{M}$ sodium acetate, $\mathrm{pH} 6.7,2.5 \mathrm{mM}$ EDTA, $50 \mu \mathrm{g} \mathrm{ml}^{-1}$ calf thymus DNA) and $200 \mu$ l of phenol. DNA was precipitated with ethanol, resuspended in the loading solution and run on gels as described above for primer extensions. Samples were run alongside a $\mathrm{G}+\mathrm{A}$ sequencing reaction performed according to the method of Maxam and Gilbert (1980).

\section{Acknowledgements}

We are grateful to I. Derré and T. Msadek for their advice, help and fruitful discussions. We thank A. Edelman for correcting this manuscript. This work was supported by research funds from the Institut Pasteur, Centre National de Recherche Scientifique and Universite Paris 7. J.V. was the recipient of a fellowship from the Ministère de l'Education Nationale, de la Recherche et de la Technologie. 


\section{References}

Blondelet-Rouault, M.H., Weiser, J., Lebrihi, A., Branny, P., and Pernodet, J.L. (1997) Antibiotic resistance gene cassettes derived from the omega interposon for use in E. coli and Streptomyces. Gene 190: 315-317.

Bourn, W.R., and Babb, B. (1995) Computer assisted identification and classification of streptomycete promoters. Nucleic Acids Res 23: 3696-3703.

Bradford, M.M. (1976) A rapid and sensitive method for the quantitation of microgram quantities of protein utilizing the principle of protein-dye binding. Anal Biochem 72: 248254.

Bucca, G., Hindle, Z., and Smith, C.P. (1997) Regulation of the dnaK operon of Streptomyces coelicolor A3(2) is governed by HspR, an autoregulatory repressor protein. $J$ Bacteriol 179: 5999-6004.

Cohen, S.N., Chang, A.C., and Hsu, L. (1972) Nonchromosomal antibiotic resistance in bacteria: genetic transformation of Escherichia coli by R-factor DNA. Proc Natl Acad Sci USA 69: 2110-2114.

de Crecy-Lagard, V., Servant-Moisson, P., Viala, J., Grandvalet, C., and Mazodier, P. (1999) Alteration of the synthesis of the Clp ATP-dependent protease affects morphological and physiological differentiation in Streptomyces. Mol Microbiol 32: 505-517.

Derré, I., Rapoport, G., and Msadek, T. (1999) CtsR, a novel regulator of stress and heat shock response, controls $c / p$ and molecular chaperone gene expression in gram-positive bacteria. Mol Microbiol 31: 117-131.

Duchêne, A.M., Kieser, H.M., Hopwood, D.A., Thompson, C.J., and Mazodier, P. (1994) Characterization of two groEL genes in Streptomyces coelicolor A3(2). Gene 144: 97-101.

Fischer, G., Wenner, T., Decaris, B., and Leblond, P. (1998) Chromosomal arm replacement generates a high level of intraspecific polymorphism in the terminal inverted repeats of the linear chromosomal DNA of Streptomyces ambofaciens. Proc Natl Acad Sci USA 95: 14296-14301.

Frees, D., and Ingmer, H. (1999) ClpP participates in the degradation of misfolded protein in Lactococcus lactis. Mol Microbiol 31: 79-87.

Gerth, U., Krüger, E., Derré, I., Msadek, T., and Hecker, M. (1998) Stress induction of the Bacillus subtilis clpP gene encoding a homologue of the proteolytic component of the Clp protease and the involvement of ClpP and CIpX in stress tolerance. Mol Microbiol 28: 787-802.

Gibson, T.J. (1984) Studies on the Epstein-Barr virus genome. PhD Thesis, Cambridge University, Cambridge, UK.

Gottesman, S., and Maurizi, M.R. (1992) Regulation by proteolysis: energy-dependent proteases and their targets. Microbiol Rev 56: 592-621.

Gottesman, S., Roche, E., Zhou, Y., and Sauer, R.T. (1998) The ClpXP and ClpAP proteases degrade proteins with carboxy-terminal peptide tails added by the SsrA-tagging system. Genes Dev 12: 1338-1347.

Gralla, J.D. (1991) Transcriptional control - lessons from an E. coli promoter data base. Cell 66: 415-418.

Grandvalet, C., Servant, P., and Mazodier, P. (1997)
Disruption of $h s p R$, the repressor gene of the dnaK operon in Streptomyces albus G. Mol Microbiol 23: 77-84.

Grandvalet, C., de Crecy-Lagard, V., and Mazodier, P. (1999) The CIpB ATPase of Streptomyces albus $\mathrm{G}$ belongs to the HspR heat shock regulon. Mol Microbiol 31: 521-532.

Grimaud, R., Kessel, M., Beuron, F., Steven, A.C., and Maurizi, M.R. (1998) Enzymatic and structural similarities between the Escherichia coli ATP- dependent proteases, ClpXP and ClpAP. J Biol Chem 273: 12476-12481.

Hopwood, D.A., Bibb, M.J., Chater, K.F., Kieser, T., Bruton, C.J., Kieser, H.M., et al. (1985) Genetic Manipulation of Streptomyces: a Laboratory Manual. Norwich: The John Innes Foundation.

Jenal, U., and Fuchs, T. (1998) An essential protease involved in bacterial cell-cycle control. EMBO $J$ 17: 5658-5669.

Jones, R.L.D., Jaskula, J.C., and Janssen, G.R. (1992) In vivo translational start site selection on leaderless mRNA transcribed from the Streptomyces fradiae aph gene. $J$ Bacteriol 174: 4753-4760.

Keiler, K.C., Waller, P.R., and Sauer, R.T. (1996) Role of a peptide tagging system in degradation of proteins synthesized from damaged messenger RNA. Science 271: 990993.

Kessel, M., Maurizi, M.R., Kim, B., Kocsis, E., Trus, B.L., Singh, S.K., and Steven, A.C. (1995) Homology in structural organization between $E$. coli ClpAP protease and the eukaryotic $26 \mathrm{~S}$ proteasome. J Mol Biol 250: 587594.

Kroh, H.E., and Simon, L.D. (1990) The ClpP component of Clp protease is the sigma 32-dependent heat shock protein F21.5. J Bacteriol 172: 6026-6034.

Laemmli, U.K. (1970) Cleavage of structural proteins during the assembly of the head of bacteriophage T4. Nature 227: 680-685.

Maurizi, M.R., Clark, W.P., Kim, S.H., and Gottesman, S. (1990) ClpP represents a unique family of serine proteases. J Biol Chem 265: 12546-12552.

Maxam, A.M., and Gilbert, W. (1980) Sequencing endlabeled DNA with base-specific chemical cleavages. Methods Enzymol 65: 499-560.

Mazodier, P., Petter, R., and Thompson, C. (1989) Intergeneric conjugation between Escherichia coli and Streptomyces species. J Bacteriol 171: 3583-3585.

Mhammedi-Alaoui, A., Pato, M., Gama, M.J., and Toussaint, A. (1994) A new component of bacteriophage $\mathrm{Mu}$ replicative transposition machinery: the Escherichia coli ClpX protein. Mol Microbiol 11: 1109-1116.

Msadek, T., Dartois, V., Kunst, F., Herbaud, M.L., Denizot, F., and Rapoport, G. (1998) ClpP of Bacillus subtilis is required for competence development, motility, degradative enzyme synthesis, growth at high temperature and sporulation. Mol Microbiol 27: 899-914.

Mullis, K.B., and Faloona, F.A. (1987) Specific synthesis of DNA in vitro via a polymerase-catalyzed chain reaction. Methods Enzymol 155: 335-350.

Murakami, T., Holt, T.G., and Thompson, C.J. (1989) Thiostrepton-induced gene expression in Streptomyces lividans. J Bacteriol 171: 1459-1466.

Muth, G., Nubbaumer, B., Wohlleben, W., and Pühler, A. (1989) A vector system with temperature-sensitive replication 
for gene disruption and mutational cloning in streptomycetes. Mol Gen Genet 219: 341-348.

Østeras, M., Stotz, A., Schmid Nuoffer, S., and Jenal, U. (1999) Identification and transcriptional control of the genes encoding the Caulobacter crescentus CIpXP protease. $J$ Bacteriol 181: 3039-3050.

Porankiewicz, J., Wang, J., and Clarke, A.K. (1999) New insights into the ATP-dependent Clp protease: Escherichia coli and beyond. Mol Microbiol 32: 449-458.

Puglia, A.M., Vohradsky, J., and Thompson, C.J. (1995) Developmental control of the heat shock stress regulon in Streptomyces coelicolor. Mol Microbiol 17: 737-746.

Redenbach, M., Kieser, H.M., Denapaite, D., Eichner, A., Cullum, J., Kinashi, H., and Hopwood, D.A. (1996) A set of ordered cosmids and a detailed genetic and physical map for the $8 \mathrm{Mb}$ Streptomyces coelicolor A3(2) chromosome. Mol Microbiol 21: 77-96.

Roche, E.D., and Sauer, R.T. (1999) SsrA-mediated peptide tagging caused by rare codons and tRNA scarcity. EMBO $J$ 18: 4579-4589.

Saiki, R.K., Gelfand, D.H., Stoffel, S., Scharf, S.J., Higuchi, R., Horn, G.T., et al. (1988) Primer-directed enzymatic amplification of DNA with a thermostable DNA polymerase. Science 239: 487-491.

Sanger, F., Nicklen, S., and Coulson, A.R. (1977) DNA sequencing with chain-terminating inhibitors. Proc Natl Acad Sci USA 74: 5463-5467.

Schweder, T., Lee, K.H., Lomovskaya, O., and Matin, A. (1996) Regulation of Escherichia coli starvation sigma factor $\left(\sigma^{\mathrm{s}}\right)$ by CIpXP protease. J Bacteriol 178: 470-476.

Servant, P., Grandvalet, C., and Mazodier, P. (2000) The RheA repressor is the thermosensor of the HSP18 heat shock response in Streptomyces albus. Proc Natl Acad Sci USA 97: 3538-3543.

Simon, R., Priefer, U., and Pühler, A. (1983) A broad host range mobilization system for in vivo genetic engineering: transposon mutagenesis in Gram-negative bacteria. Bio/ Technology 1: 784-791.

Strohl, W.R. (1992) Compilation and analysis of DNA sequences associated with apparent streptomycete promoters. Nucleic Acids Res 20: 961-974.
Studier, F.W., and Moffatt, B.A. (1986) Use of bacteriophage T7 RNA polymerase to direct selective high-level expression of cloned genes. J Mol Biol 189: 113-130.

Tobias, J.W., Shrader, T.E., Rocap, G., and Varshavsky, A. (1991) The N-end rule in bacteria. Science 254: 13741377.

Tu, G.F., Reid, G.E., Zhang, J.G., Moritz, R.L., and Simpson, R.J. (1995) C-terminal extension of truncated recombinant proteins in Escherichia coli with a 10Sa RNA decapeptide. $\checkmark$ Biol Chem 270: 9322-9326.

Turgay, K., Hahn, J., Burghoorn, J., and Dubnau, D. (1998) Competence in Bacillus subtilis is controlled by regulated proteolysis of a transcription factor. EMBO J 17: 67306738.

Volff, J.N., and Altenbuchner, J. (1998) Genetic instability of the Streptomyces chromosome. Mol Microbiol 27: 239246.

Wang, J., Hartling, J.A., and Flanagan, J.M. (1997) The structure of ClpP at $2.3 \AA$ resolution suggests a model for ATP-dependent proteolysis. Cell 91: 447-456.

Wawrzynow, A., Wojtkowiak, D., Marszalek, J., Banecki, B., Jonsen, M., Graves, B., et al. (1995) The ClpX heat-shock protein of Escherichia coli, the ATP-dependent substrate specificity component of the ClpP-ClpX protease, is a novel molecular chaperone. EMBO J 14: 1867-1877.

Wehmeier, U.F. (1995) New multifunctional Escherichia coliStreptomyces shuttle vectors allowing blue-white screening on XGal plates. Gene 165: 149-150.

Wojtkowiak, D., Georgopoulos, C., and Zylicz, M. (1993) Isolation and characterization of ClpX, a new ATPdependent specificity component of the Clp protease of Escherichia coli. J Biol Chem 268: 22609-22617.

Woo, K.M., Chung, W.J., Ha, D.B., Goldberg, A.L., and Chung, C.H. (1989) Protease Ti from Escherichia coli requires ATP hydrolysis for protein breakdown but not for hydrolysis of small peptides. J Biol Chem 264: 2088-2091.

Yanisch-Perron, C., Vieira, J., and Messing, J. (1985) Improved M13 phage cloning vectors and host strains: nucleotide sequences of the M13mp18 and pUC19 vectors. Gene 33: 103-119. 